\title{
ROAD TRAFFIC NOISE PREDICTION MODELS: A REVIEW
}

\author{
H.N. RAJAKUMARA ${ }^{1}$ \& R.M. MAHALINGE GOWDA ${ }^{2}$ \\ ${ }^{1}$ Department of Civil Engineering, Adhiyamaan College of Engineering, Hosur, Tamil Nadu, India. \\ ${ }^{2}$ Department of Civil Engineering, P.E.S. College of Engineering, Mandya, Karnataka, India.
}

\section{ABSTRACT}

The environmental quality of our cities is gradually degrading by an incessant growth in the number of vehicles and the ever expanding road network, resulting in the increase of road traffic noise. Managing road traffic noise is a challenging task for environmental managers and urban planners. Urban planners often have to rely on road traffic noise prediction models for their assessment. A critical review of various traffic noise studies and the number of traffic noise prediction models cited in literature reveals that they describe the temporal and spatial distribution of traffic noise. Most of these models are either deterministic or statistical in nature. This article presents a critical review of some of these models.

Keywords: ASJ model and GIS model, CoRTN model, FHWA model, FHWA TNM model, RLS90, Stop-and-go model.

\section{INTRODUCTION}

Road traffic noise has become a major concern of communities living in the vicinity of major highway corridors. It is causing more disturbances to people than any other sources. Moreover, this menace to health and quality of life has been increasing over the last two decades for number of reasons [1]. The most important cause is of the number of road vehicles, and consequently, increases in the density of road traffic. The construction of multi-lane motorways is going on at increasing rates in most developed countries and even in many developing nations during last few decades, allowing large volume of traffic to travel at a sustained speed. The next most important cause of noise on the roads is the speed of traffic. As a general rule, faster the traffic moves, greater is the volume of noise [2]. Surveys conducted in many countries have shown that traffic noise is one of the principal environmental nuisances in urban areas, and most of the countries have their own traffic noise prediction model according to the traffic and environmental conditions.

Traffic noise prediction models are required as aids in the design of highways and other roads and sometimes in the assessment of existing or envisaged changes in traffic noise conditions. They are commonly needed to assess noise levels set by government authorities. Environmental laws require the Environmental Impact Statement (EIS) to take into account the effect of the proposed noise on all existing and potential elements of the environment, besides statutory criteria. This calls for a variety of descriptors and criteria. Special descriptors are sometimes required for the assessment of complaints about road traffic noise [3].

Traffic noise prediction models are required for use by five main groups, viz.,

- Roadway engineers, who check designs for compliance with statutory noise constraints and determine any need for screens or additional spacing between road and buildings.

- Acoustical engineers for fine work such as architectural and more general applications.

- Expert witnesses in civil or criminal courts or other officials enquiries, whose opinion is usually required in addition to an assessment of any statutory requirements.

- Acoustic specialists, who prepare the acoustic section of EISs.

- Acoustic consultants, engaged by clients perhaps adversely affected by road traffic noise. Such cases preliminarily require remedies and recommendations. Models may be used to 
ascertain whether measured noise is consistent with appropriate design or, in a few cases, statutory conditions.

As per reviewed literature, it is observed that various traffic noise studies were reported and a number of traffic noise prediction models have been developed. To the best knowledge of the authors, the more popular ones include the CoRTN model in UK, the Federal Highway Administration (FHWA) model in USA, the RLS90 model in Germany, the OAL model in Austria, the Statens Planverk 48 model in Scandinavia, the EMPA model in Switzerland, the ASJ model in Japan and the GIS model in China. A critical review of some of these models is discussed in this article.

\section{TRAFFIC NOISE PREDICTION MODELS}

The prediction models considered here represent national responses to the noise pollution concerns, which arose from the great increase in automobile ownership after World War II and also from the current interest in environmental matters generally [3]. The FHWA Traffic Noise Model Version 1.0 is a development of the earlier FHWA Traffic Noise Model. With that exception, the models considered here had parallel independent development, albeit with some theoretical interaction.

Most current models assume point sources, although some assume line sources. Rathe [4] found an analytical solution of the problem for incoherent point sources in a line with given spacing and given angle of view. The Japanese model (the ASJ Model, 1993) adopts this form. Steele [5] gave a more general solution. This solution admits of roads of any shape with either line or multiple point sources, but with time and not distance-determined spacing. This allows for acceleration and braking as well as steady flow. Directivity may be accommodated in several models. Recent models incorporate a propagation section, even though there is a current international standard [6] for calculation of outdoor sound attenuations. Although individual in their detail, these propagation equations are generally similar to that of Maekawa [7].

A number of European models incorporate submodels for the prediction of traffic flow itself, whereas American and British models assume that such inputs are to be had from other sources [3]. In developing countries, traffic noise studies are relatively less when compared to the developed nations. Among the developing countries, China is in progress in conducting traffic noise studies. Recently, it has developed its own traffic noise prediction model with GIS applications [2]. India has now slowly started its efforts in developing its own traffic noise prediction model [8].

\subsection{The FHWA model}

In response to the widely recognized shortcomings of existing highway noise prediction methodologies, Barry and Reagan of the US FHWA developed an in-house model in 1979 [9] by considering those areas that had not been addressed by the National Cooperative High-way Research Program (NCHRP) and Transportation Systems Centre (TSC) models of USA. The model was published in the report FHWA-RD-77-108 which included a programmable calculator program. This program was further developed separately under the title STAMINA in several successive versions. The FHWA model calculates noise level through a series of adjustments to a reference sound level. The reference sound level is the energy mean emission level which is determined through field measurements of individual vehicle. Adjustment are then made to this level to account for traffic flow, distance of receivers from the roadways, finite length roadways, ground cover, and shielding effects.

The model assumes point source traveling at constant speed. The authors compared predicted A-weighted sound pressure levels with data collected in a program known as the Four State Noise 
Inventory [10]. The accuracy of the method was found to depend on the distance of the receiver from the source, and also on vehicle composition. In comparing some Florida traffic with the national noise emission levels [10], the mean errors were found to be $-0.05,-0.95$, and $-1.3 \mathrm{~dB}(\mathrm{~A})$ at horizontal distances of 15,30 , and $60 \mathrm{~m}$, respectively. The corresponding standard deviations were $1.64,1.82$, and $2.39 \mathrm{~dB}(\mathrm{~A})$. However, comparison made with noise emission levels of Florida only gave mean errors of $+0.58,-2.3$, and $-0.57 \mathrm{~dB}(\mathrm{~A})$ with slightly smaller standard deviations. They gave some examples of insertion loss calculations for a barrier. In one case, the predicted loss was $7.2 \mathrm{~dB}(\mathrm{~A})$, whereas the measured was $10 \mathrm{~dB}(\mathrm{~A})$. The STAMINA program allows a convenient adjustment to the reference emission levels. Junf et al. [11] reported an adaptation for Ontario with fair success, and that about $4 \%$ of trucks were excessively noisy and caused an upward increase of $0.5-1.0 \mathrm{~dB}(\mathrm{~A})$ in the reference level. The authors thought this comparable with observations made elsewhere.

This procedure is strictly applicable to straight roads and vehicles of constant speed, but methods are incorporated for the use of segments to simulate curved roads and multiple lanes.

Three major assumptions were made in this model, viz.,

1. The vehicles are adequately represented by acoustic point sources.

2. Emissions levels within groups (automobile, medium, and heavy trucks) are normally distributed (although they are skewed to the high side).

3. Propagation losses are adequately represented by distance effects.

In the original FHWA-RD-77-108 format, standardized reference energy mean emission levels (REMEL) for three classes - automobile, medium trucks (MT), and heavy trucks (HT) - were employed. They are expressed as sound pressure levels at $15 \mathrm{~m}$ from the sources as functions of the speed of the vehicle. In addition to the mean levels, account was taken of the statistical distribution at each speed for each class.

All of these adjustments are related by the following equation.

$$
\begin{aligned}
L_{\mathrm{eq}(h) i}= & L_{0}+0.115 \sigma^{2}+10 \log \left[N_{i} \pi D_{\mathrm{o}} / S_{i} T\right]+10 \log \left[D_{0} / D\right]^{1+a} \\
& +10 \log \left[\psi \alpha\left(\phi_{1}, \phi_{2}\right) / \pi\right]+\Delta s,
\end{aligned}
$$

where $L_{\mathrm{eq}(h) i}$ is the hourly equivalent sound pressure level for $i$ th class of vehicle, $L_{0}$ is the hourly mean sound pressure level at the reference distance, $N_{i}$ is the vehicles volume for $i$ th class of vehicle in passenger car unit per hour, $S_{i}$ is the mean speed of $i$ th class of vehicle in $\mathrm{km}$ per hour, $D$ is the perpendicular distance from the center line of the traffic lane to the receiver, $D_{\mathrm{o}}$ is the reference distance from the center line of road to the observer, $T$ is the time period over which $L_{\text {eq }}$ is computed $(1 \mathrm{~h}), \alpha$ is a site parameter $(0<\alpha<1), \phi_{1}$ and $\phi_{2}$ are the angles from the perpendicular of the limits of the observer's view of a section of the road way, and $\Delta s$ is the excess attenuation due to barriers, buildings, wood, etc.

Attenuation due to shielding is an important mechanism by which road traffic noise levels are lowered. Shielding can be provided by different types of noise barriers such as berms, walls, large buildings, etc. Barriers affect sound propagation by interrupting the sound waves and creating an acoustic shadow zone. The FHWA model expresses the attenuation by noise barriers as a function of the Fresnel number, the barrier shape, and the barrier length. The acoustic phenomenon governing barrier attenuation is known as Fresnel diffraction which analytically defines the amount of the acoustic energy loss encountered when sound waves are required to travel over and around a barrier. 
The proposed equation for calculating noise attenuation due to thin barriers is given by:

$$
\Delta L_{\mathrm{S}}=10 \log \left[\frac{1}{\phi_{\mathrm{R}}-\phi_{\mathrm{L}}} \int_{\phi_{\mathrm{L}}}^{\phi_{R}} 10^{-\Delta i / 10} \mathrm{~d} \phi\right],
$$

where $\Delta L_{\mathrm{S}}$ is the attenuation for the $i$ th class of vehicle, and $\phi_{\mathrm{R}}$ and $\phi_{\mathrm{L}}$ are the angles measured from the perpendicular to the right and left ends of the barriers, respectively.

The total noise due to all types of vehicles can be calculated by:

$$
L_{\mathrm{eqT}}=\sum_{\text {Sum }}^{\mathrm{dB}(\mathrm{A})} L_{\mathrm{eq}}(C+M T+H T)=10 \log \left(10^{L_{\mathrm{eqC}}}+10^{L_{\mathrm{eqMT}}}+10^{L_{\mathrm{eqHT}}}\right),
$$

where $L_{\text {eqT }}$ is the total equivalent traffic noise due to all class of vehicles, $C$ is the equivalent noise for car, $M T$ is the equivalent noise for medium trucks, and $H T$ is the equivalent noise for heavy trucks.

As mentioned before, the FHWA model computes predicted sound levels through a series of adjustments to a reference sound level. These adjustments depend on traffic patterns, topography, and roadway geometry, and are available from different figures and tables. Various procedures have been developed for implementing the FHWA model. These include a manual method, nomographs, handled calculators, and computer programming. A more powerful version of the model evolved in the form of STAMINA 1.0 (FHWA, 1979) program. This program was coordinate-based and could simultaneously consider $L_{\mathrm{eq}}$ values for multiple receivers and complex roadway barrier geometry. Enhancements to this program resulted in the latest program for highway noise analysis, STAMINA 2.0. This version of FHWA model is used almost exclusively for highway noise analysis in USA and in many other countries as well.

\subsection{The FHWA TNM model of version 1.0}

The FHWA TNM E9, Version 1 was introduced by Anderson et al. [12]. Although derived from the STAMINA 2.0 program, it has many substantial improvements. It admits of imports from CAD programs and STAMINA 2.0. Improvements on earlier models include provision for acceleration, stop signs, traffic signals, etc. Another improvement is the provision for the input of user-defined vehicles using their REMEL data. This is represented in the form of one-third octave band spectra.

The general REMEL equation is a function of speed and frequency, as given below:

$$
\begin{aligned}
L_{\mathrm{E}}(s, f)= & 10 \log _{10}\left[10^{\left(C+\Delta E_{\mathrm{c}}\right) / 10}+\left(S^{A / 10}\right)\left(10^{\left(B+\Delta E_{\mathrm{b}}\right) / 10}\right)\right]-(K 1+K 2 . s)+D 1+D 2 . s \\
& \left.+(E 1+E 2 . s) \log _{10} f+(F 1+F 2 . s) \log _{10} f\right)^{2}+(G 1+G 2 . s)\left(\log _{10} f\right)^{3} \\
& +(H 1+H 2 . s)\left(\log _{10} f\right)^{4}+(I 1+I 2 . s)\left(\log _{10} f\right)^{5}+(J 1+J 2 . s)\left(\log _{10} f\right)^{6},
\end{aligned}
$$

where $\mathrm{A}$ is the slope of the tyre/pavement portion of the regression curve, $B+\Delta E b$ is the height of the tyre/pavement portion, $C+\Delta E c$ is the height of the engine/exhaust portion, $D 1+J 2$ are constants of the sixth-order polynomial fit curve for the one-third spectra, and $K 1$ and $K 2$ give the A-weighted level $L(s)$, instead of $L(s, f)$.

The sound pressure levels are then apportioned between the sub-sources, tyre/road and engine/exhaust according to frequency, thus:

$$
\operatorname{SSR}(f)=L+[1-L-M]\left[1+\mathrm{e}^{(N \log f+p)}\right]^{Q},
$$


where $L$ is the sub-source ratio at lower frequencies, $1-M$ is the ratio at high frequencies, and $N$, $P$, and $Q$ are constants.

\subsection{The CoRTN model}

This model was published by the Department of the Environment and Welsh Office in UK in 1975 by Delany et al. [13]. It is used as an aid to road design, and also for the determination of entitlements to the sound insulation of private dwellings at public expanse under the British Land Compensation Act. This latter influenced the choice of $L_{10}$ as the index of noise. CoRTN is distinguished by its extensive use of curve fitting between empirical data, even when this was known not to conform to theory.

This model assumes a line source and constant speed traffic, and in Britain, it is the sole instrument for the assessment of road traffic environmental impacts by road authorities. Calculation of Road Traffic Noise [14] (CoRTN) was replaced by a more convenient Predicting Road Traffic Noise [15] which followed Delany et al. [13] rationale for the procedure. These authors reported that for the range $50-54.9 \mathrm{~dB}(\mathrm{~A})$, the mean difference between their predicted and measured levels was +1.4 $\mathrm{dB}(\mathrm{A})$. On the other hand, between 80 and $84.9 \mathrm{~dB}(\mathrm{~A})$, the mean error was $-1.2 \mathrm{~dB}(\mathrm{~A})$. That is, CoRTN underestimated high levels and overestimated low levels. Samuels \& Saunders [16] observed significant differences in the model with Australian vehicles, depending upon the prevailing conditions. The mean overestimation was $0.7 \mathrm{~dB}(\mathrm{~A})$ for free field conditions and $1.7 \mathrm{~dB}(\mathrm{~A})$, in front of facades with corresponding standard deviations 1.8 and $2.5 \mathrm{~dB}(\mathrm{~A})$. Some users came to depend on CoRTN for applications for which it was not strictly valid, for which Steele [5] found even greater under or overestimates by as much as $+12.5 \mathrm{~dB}(\mathrm{~A})$ in one case. Computer programs based on CoRTN were written for various authorities, for example, in Weatherall [17]. This model has applicability to long line of free flowing rush hour traffic or train at a distance from the observer. It is less suitable for situations where the distance is not great in relation to the inter-vehicular spacing, or when the spacing is very even or uneven.

First, the basic noise level at a reference distance of $10 \mathrm{~m}$ away from the nearside carriageway edge is obtained from the traffic flow, the speed of traffic, the composition of the traffic, the gradient of the road, and the road surface.

The noise emission level equation for $L_{10}$ is given as:

$$
L_{10}=L_{0}+A_{\mathrm{HV}}+A_{\mathrm{D}}+A_{\mathrm{G}}+A_{\mathrm{GC}}+A_{\mathrm{a}}+A_{\mathrm{B}},
$$

where $L_{0}$ is the basic noise emission level and,

$$
L_{0}=42.2+10 \log _{10} q \text {. }
$$

$A_{\mathrm{HV}}$ is the adjustment for mean traffic speed and percentage of heavy vehicles:

$$
A_{\mathrm{HV}}=33 \log _{10}(V+40+500 / V)+10 \log _{10}(1+5 \mathrm{P} / V)-68.8,
$$

where $V$ is the traffic speed that depends upon the road classification as specified by CoRTN model, and $P$ is the percentage of heavy vehicles.

$$
P=100 f / q \text {. }
$$

where $f$ is the hourly flow of heavy vehicles, $q$ is the hourly flow of all vehicles, and $A_{\mathrm{D}}$ is the distance adjustment.

$$
A_{\mathrm{D}}=-10 \log _{10}\left(d^{*} / 13.5\right),
$$

where $d^{*}$ is the shortest slant distance from the effective source position, in $\mathrm{m}$. 


$$
d^{*}=\left[(d+3.5)^{2}+h^{2}\right]^{1 / 2}
$$

where $d$ is the shortest horizontal distance from the edge of the nearside carriageway to the reception point, $h$ is the height of reception point relative to the source line at the point where the slant line intersects the source line at the effective source position, $S$.

$A_{\mathrm{G}}$ is the adjustment for gradient $G$ :

$$
A_{\mathrm{G}}=[0.73+(2.3-1.15 P / 100) P / 100] \times G,
$$

where $G$ is the gradient of roadway.

$A_{\mathrm{GC}}$ is the adjustment for ground cover:

$$
\begin{array}{ll}
A_{\mathrm{GC}}=5.2 I \log _{10}(6 H-1.5 / d+3.5) & \text { for } 0.75 \leq H<(d+5) / 6, \\
A_{\mathrm{GC}}=0 & \text { for } H \geq(d+5) / 6, \\
A_{\mathrm{GC}}=5.2 I \log _{10}(3 / d+3.5) & \text { for } H<0.75,
\end{array}
$$

where $H$ is the mean height of propagation, $I$ is the proportion of absorbing ground between the edge of the nearside carriageway and the segment boundaries leading to the reception point $\mathrm{R}$.

$A_{\mathrm{a}}$ is the correction for angle of view:

$$
A_{\mathrm{a}}=10 \log _{10}(\theta / 180)
$$

where $\theta$ is the angle of view.

CoRTN also contains a calculation of the attenuation due to thin barriers. Instead of calculating the attenuations frequency by frequency, an A-weighted attenuation is attempted. No allowance is made for differences between spectra. The potential barrier correction is calculated as a function of path difference $(\delta)$. CoRTN model gives a polynomial expression for potential barrier correction as:

$$
A_{\mathrm{B}}=a_{0}+a_{1} X+a_{2} X^{2}+a_{3} X^{3}+\cdots+a_{n} X^{n}
$$

where

$$
X=\log _{10} S
$$

in which $S$ is the path length difference, in meters, between the direct and diffracted rays, and $a_{0}$ to $a_{n}$ are constants.

\subsection{The stop-and-go model}

This model was developed for central part of Bangkok by Urban Transport Department in 1997 by Pamanikabud and Tharasawatpipat [18]. The research focuses toward formulating an empirical model of interrupted traffic flow in Bangkok using two analytical approaches. The first being the single model analysis, and the second, the separated model or dual model analysis. In this study, several parameters that are considered to have possible influences on the interrupted traffic flow noises were measured at the study sites. Then, these were tested for their correlation with traffic noise levels. The parameters consisted of vehicle volume which is classified into different vehicle types appearing on the Bangkok roadways, average spot speed of vehicles in the traffic stream, road width, distance from curb to building façade, and distance to the nearest intersections. In the analysis of data, these parameters also were separated into nearside and farside roadway parameters. In this study, the distances to building façade were applied by two parameters. The first was the distance from observer to nearside building facade, and second, the distance from observer to farside building facade. Geometric mean of the roadway cross-section was introduced as one of the parameters in the 
analysis of the stop-and-go traffic noise model. Tests were conducted to determine the correlation of various parameters with the traffic noise level in $L_{\mathrm{eq}}$ as well as the collinearity among these parameters if they existed. The sets of parameters which correlated highly with $L_{\mathrm{eq}}$ were used further as input in multiple regression analysis. Stepwise analysis technique was adopted in the multiple regression analysis processes of the study.

Individual noise characteristics at an overall mean vehicle speed was used to identify the proportional weighting scale of the noise levels generated per unit of each vehicle type in relation to an automobile unit. The overall spot speed range obtained from the field survey data of this research together with the value for overall mean vehicle speed (of $33 \mathrm{~km} / \mathrm{h}$ ) were superimposed onto the vehicle noise characteristics. As there are many vehicle types that are in use in Bangkok, these were classified into seven groups based on the similarities in their noise level characteristics within the speed range that was observed in this study. From this analysis, the proportional weighting scale of the noise levels generated by each vehicle class was calculated. With this information, the traffic of traffic used in the model then could be described in terms of the noise generating ratio of each vehicle type in comparison with automobiles in Bangkok's urban traffic.

The equation for traffic volume can be given as follows:

$$
\begin{aligned}
& \text { Volume of traffic }\left(V_{n} \text { or } V_{f}\right)=\mathrm{AU}+1.04(\mathrm{LT})+1.12(\mathrm{MT}+\mathrm{TT}) \\
& +1.14(\mathrm{HT})+1.09(\mathrm{MC}+\mathrm{BU}+\mathrm{MB}),
\end{aligned}
$$

where AU stands for automobile; HT, heavy truck; LT, light truck; MC, motorcycles; MT, medium truck; BU, bus, and TT for tuk-tuk.

In order to validate the model, another set of data was collected from 10 new locations in the central part of Bangkok. The results indicated that the mean differences between measured and predicted values of $0.09192 \mathrm{~dB}(\mathrm{~A})$ and $0.02219 \mathrm{~dB}(\mathrm{~A})$ occur in the goodness-of-fit test with 127 data sets for acceleration and deceleration lanes, respectively.

\subsubsection{The single model analysis}

This approach was applied first to build a single stop-and-go traffic flow model. This can be applied to both sides of an urban roadway. The model developed in this study is given as:

$$
L_{\text {eq }}=71.05+0.10 S_{\mathrm{n}}+0.95 \log V_{\mathrm{n}}+0.04 S_{\mathrm{f}}+0.015 \log V_{\mathrm{f}}-0.111 D_{\mathrm{g}},
$$

where $L_{\mathrm{eq}}$ is the equivalent traffic noise level in one hour in $\mathrm{dB}(\mathrm{A}), S_{\mathrm{n}}$ and $S_{\mathrm{f}}$ are the mean speed of traffic on nearside and farside of observer in $\mathrm{km} / \mathrm{h}$, and $V_{\mathrm{n}}$ and $V_{\mathrm{f}}$ are the volume of traffic for nearside and farside of traffic in vehicles per hour.

$D_{\mathrm{g}}$ is the geometric mean of roadside section:

$$
D_{\mathrm{g}}=\sqrt{D_{\mathrm{f}} \times D_{\mathrm{n}}},
$$

where $D_{\mathrm{n}}$ and $D_{\mathrm{f}}$ are the distance from the observer to center line of nearside and farside road way, in $\mathrm{m}$.

2.4.2 The separate lane model analysis

This approach acknowledges the difference in traffic noise characteristics between acceleration lane and deceleration lane of both sides of the urban road when vehicles leave and intersect on a green traffic light and come to stop on red traffic light. The acceleration lane model was built using data generated from the noise level meter placed on the sidewalk near the acceleration lane on the 
roadway when the traffic leaves the intersection. These models for accelerated and decelerated situations are as follows.

Acceleration lane interrupted traffic noise model:

$$
L_{\mathrm{eq}}=56.91+0.09 S_{\mathrm{n}}(a)+5.22 \log V_{\mathrm{n}}(a)+0.04 S_{\mathrm{f}}(a)+0.02 \log V_{\mathrm{f}}(a)-0.061 D_{\mathrm{g}}(a) .
$$

Deceleration lane interrupted traffic noise model:

$$
L_{\mathrm{eq}}=71.12+0.07 S_{\mathrm{n}}(b)+0.42 \log V_{\mathrm{n}}(b)+0.08 S_{\mathrm{f}}(b)+0.44 \log V_{\mathrm{f}}(b)-0.061 D_{\mathrm{g}}(b) .
$$

\subsection{The ERTC model}

This model was developed by the Environmental Research and Training Centre (ERTC) of Thailand for environmental impact assessment [19]. In this model, vehicles were classified into two groups and the average stationary noise level of each group was then determined by measurement of many vehicles. The power level of each group was determined by measuring the noise level of running vehicles.

The equivalent sound level ' $L_{\mathrm{eq}}$ ' observed at a certain receiving point is given by,

$$
L_{\mathrm{eq}}=\mathrm{PWL}-10 \log 2 l d+L_{\mathrm{d}}+L_{\mathrm{g}}
$$

where PWL is A-weighted energy average power level of vehicle, $\mathrm{dB}(\mathrm{A}), l$ is the distance from a traffic line to receiving point, $\mathrm{m}, L_{\mathrm{d}}$ and $L_{\mathrm{g}}$ are the correction value for distance and diffraction attenuation, $\mathrm{dB}(\mathrm{A})$, and $d$ is the average distance between front of vehicles, $\mathrm{m}$.

$$
d=1000 \times V / Q
$$

where $V$ is the average speed of vehicles, $\mathrm{km} / \mathrm{h}$ and $Q$ is the traffic volume, Vehicles $/ \mathrm{h}$.

The energy power level of vehicles is given by:

For large vehicle group:

$$
\mathrm{PWL}=75.1+20.4 \log V .
$$

For small vehicle group:

$$
\mathrm{PWL}=67.8+20.4 \log V .
$$

The average noise level of large group of vehicles is about $7.3 \mathrm{~dB}(\mathrm{~A})$ higher than that of small group of vehicles and the average power level for a number of vehicles of mixed type is given as follows:

$$
\mathrm{PWL}=67.8+20.4 \log V+10 \log [(1-a)+5.37 a]-10 \log 2 l d+L_{\mathrm{d}}+L_{\mathrm{g}},
$$

where $a$ is the ratio of the large number of vehicles to the total number vehicles.

The study concluded that the accuracy of the model is sufficient for practical use and will be used for environmental impact assessment in Thailand. It has been shown that the model can be used for 2, 4, 6, 8, and 10 lane highways in cases where speed is between 30 and $140 \mathrm{~km} / \mathrm{h}$. The accuracy of the model has been shown to be within $\pm 3 \mathrm{~dB}(\mathrm{~A})$ range about $92.3 \%$ of the time and that it can predict the road traffic noise level at a distance of $1-80 \mathrm{~m}$, and at a height of 1-12 $\mathrm{m}$ from the ground.

\subsection{The RLS 90 model}

Richtlinien fur den Larmschutz an Straßen (RLS-90) (Guidelines for Noise Protection on Streets) is an anonymous legal standard for noise prediction in Germany [3]. The current 1990 issue replaced the original 1981 issue. It incorporates traffic flow design data where the actual flow is not known. 
The assessed sound pressure level for a street is given by:

$$
L_{\mathrm{T}}=L_{\mathrm{m}}+K,
$$

where $L_{\mathrm{m}}$ is the mean A-weighted level, and $K$ is the addition for increased effect of traffic light controlled intersections and other intersections.

The mean A-weighted level is given by:

$$
L_{\mathrm{m}}=10 \log \left[10_{\mathrm{m} . \mathrm{n}}^{0.1 L}+10_{\mathrm{m} . \mathrm{f}}^{0.1 L}\right],
$$

where $n$ and $f$ represent the nearer and further lanes, respectively.

For long, straight traffic streams:

The mean noise level for each lane is calculated from,

$$
L_{\mathrm{m}}=L_{\mathrm{m} . \mathrm{E}}+D_{\mathrm{s}} \perp+D_{\mathrm{BM}}+D_{\mathrm{B}},
$$

where $L_{\mathrm{m} . \mathrm{E}}$ is the emission level, $D_{\mathrm{s}} \perp$ is the attenuation due to distance and air absorption, $D_{\mathrm{BM}}$ is the attenuation due to ground and atmospheric effects, and $D_{\mathrm{B}}$ is the attenuation due to the topography and building dimensions.

The emission level is calculated by,

$$
L_{\mathrm{m} . \mathrm{E}}=L_{\mathrm{m}}{ }^{(25)}+D_{\mathrm{v}}+D_{\mathrm{StrO}}+D_{\mathrm{Stg}}+D_{\mathrm{E}}
$$

where $L_{\mathrm{m}}{ }^{(25)}$ is the A-weighted mean level, $D_{\mathrm{v}}$ is a correction for speed limits, $D_{\mathrm{StrO}}$ is a correction for road surfaces, $D_{\mathrm{Stg}}$ is a correction for rises and falls, and $D_{\mathrm{E}}$ is a correction for the absorption characteristics of building surfaces.

$$
L_{\mathrm{m}}^{(25)}=37.3+10 \log [M(1+0.082 p)],
$$

where $M$ is the standardized traffic flow according to whether the road is a Federal, State, District, or Municipal connecting roads, and $p$ is the percentage of heavy vehicles.

The value of each parameter that depends upon whether day $(6.00-22.0 \mathrm{~h})$ or night $(22.0-6.00 \mathrm{~h})$ is under consideration.

$$
\begin{gathered}
D_{\mathrm{v}}=L_{\mathrm{Pkw}}-37.3+10 \log \left\{\frac{100+\left(10^{0.1 D}-1\right) p}{100+8.23 p}\right\}, \\
L_{\mathrm{Pkw}}=27.7+10 \log \left[1+\left(0.02 v_{\mathrm{Pkw}}\right)^{3}\right], \\
L_{\mathrm{Lkw}}=23.1+12.5 \log v_{\mathrm{Lkw}}, \\
D=L_{\mathrm{Lkw}}-L_{\mathrm{Pkw}},
\end{gathered}
$$

where $v_{\mathrm{Pkw}}$ is the speed limit in the range of $30-130 \mathrm{~km} / \mathrm{h}$ for light vehicles, $v_{\mathrm{Lkw}}$ is the speed limit in the range of $30-80 \mathrm{~km} / \mathrm{h}$ for heavy vehicles, $L_{\mathrm{Pkw}}$ and $L_{\mathrm{Lkw}}$ are the corresponding mean noise levels, $L_{\mathrm{m}}{ }^{(25)}$, and $D_{\mathrm{StrO}}$ is the correction for road surface given in a table and depends upon the kind of surface and vehicle speed. It ranges from 0 to $6 \mathrm{~dB}(\mathrm{~A})$.

$$
\begin{array}{ll}
D_{\mathrm{Stg}}=0.6[G]-3 & \text { for }[G]>5 \%, \\
D_{\text {Stg }}=0 & \text { for }[G] \leq 5 \% .
\end{array}
$$

RLS 90 is unique, among the programs considered here, in having an algorithm for parking lots. The calculations are similar to those for roads. The sound emission level is calculated from,

$$
L_{\mathrm{m} . \mathrm{E}}^{*}=37+10 \log (N, n)+D_{\mathrm{P}},
$$


where $N$ is the number of vehicle movements per hour by parking spot, $N$ is the number of such parking spots, and $D_{\mathrm{P}}$ is a correction for the type of car park.

Attenuation is calculated with usual ray tracing methods. Barriers, elevated and depressed roads are treated in usual way for incoherent line sources.

\subsection{The ASJ model}

In 1975, the Acoustical Society of Japan published a method of predicting a pseudo- $L_{50}$ resulting from freely flowing road traffic. It was reported by Koyasu in 1978 [20] and up-dated by Takagi \& Yamamoto in 1993 [21]. The up-dated version contains a direct method of calculating $L_{\mathrm{eq}}$. This is termed as A-method. The ASJ model also includes an empirical method called the B-method which is valid only far from the line source.

The sound power levels for traffic stream can be calculated by:

For two classes of vehicle:

$$
L_{\mathrm{W}}=65.1+20 \log V+10 \log \left(a_{1}+4.4 a_{2}\right),
$$

where $a_{1}$ and $a_{2}$ are the proportions of light and heavy vehicles.

For three classes of vehicles:

$$
L_{\mathrm{W}}=64.7+20 \log V+10 \log \left(b_{1}+1.5 b_{2}+b_{3}\right),
$$

where $b_{1}, b_{2}$, and $b_{3}$ are constants corresponding to light, medium, and heavy vehicles.

The ASJ method admits of a precise, A-method and an empirical B-method.

A-method:

This method calculates the octave band spectra. These are derived from the band center frequencies from 63 to $4,000 \mathrm{~Hz}$ according to the equation:

$$
\mathrm{L}(f)=-10 \log \{1+(f / 2,000)\} \pm 2.5 \log (f / 1,000) .
$$

B-method:

$$
L_{\mathrm{eq}}=10 \log \left[\sum_{i=1}^{k} 10^{U_{i} / 10} \Delta t N / T\right]
$$

where, $U_{i}$ is the $i$ th subinterval of $U(f)$, the range of the propagation function at the receiver, $N$ is the traffic volume, and $T$ is the time equal to $3,600 \mathrm{~s}$.

$$
\Delta t=\Delta d / v,
$$

where $\Delta d$ is the spacing between the vehicles.

The basic propagation equation is based on Rayleigh [22], but modified to incorporate the A-weighting.

$$
L_{\mathrm{eq}}=10 \log \left[\sum 10^{L_{\mathrm{wi}} / 10} \phi_{i}^{2} / 2 \pi\right],
$$

where $L_{\mathrm{Wi}}$ is A-weighted and $\phi$ is the velocity potential.

The pseudo $L_{50}$ may be found from:

$$
L_{50}=L_{\text {eq }}+a d / 1+b,
$$

where $d$ is the vehicle spacing and $l$ is the distance from the road center to the receiver, $a$ and $b$ are constants depending on the relative elevation of the road. 


\subsection{The GIS model of China}

This GIS based road traffic noise prediction model has been developed for China in 2002 [2]. The model is developed based on local environmental conditions, vehicle types and traffic conditions in China. An integrated noise-GIS system was developed to provide general functions for noise modeling and an additional tool for noise barrier design, where a new interaction mode in 'WHAT IF Question/Explanation' format was used. Application of this system offered improvements in the efficiency and accuracy of traffic noise assessment and noise barrier design.

In this study, vehicles have been classified in to three types, namely, light cars (LC), medium trucks (MT), and heavy trucks (HT). A composite relationship was developed based on factor of acoustic equivalence between different vehicle classes. The final form of the traffic noise prediction model of the study is given by:

$$
\begin{aligned}
L_{\mathrm{eq}}= & 55.7 & & \\
& +0.12\left(V_{\mathrm{E}}-50\right)-8.06 \log V_{\mathrm{E}} & & \text { adjustment for speed } \\
& +9.97 \log Q_{\mathrm{E}} & & \text { adjustment for traffic flow } \\
& +14.38 \log \left(D_{\mathrm{o}} / D\right) & & \text { adjustment for distance } \\
& +L_{\mathrm{Gradient}} & & \text { adjustment for gradient } \\
& +a P 10 \log (D \mathrm{D} / D) & & \text { adjustment for ground absorption } \\
& +10 \log (\Delta \phi / 180) & & \text { adjustment for finite length of road segment } \\
& +L_{\mathrm{S}} & & \text { shielding adjustment. }
\end{aligned}
$$

where $L_{\mathrm{eq}}$ is the equivalent noise level, $\mathrm{dB}(\mathrm{A}), V_{\mathrm{E}}$ is the equivalent speed of traffic flow, $\mathrm{km} / \mathrm{h}, Q_{\mathrm{E}}$ is the equivalent traffic flow, Veh/h, $D_{\mathrm{o}}$ is the measurement distance for the reference noise emission level $=7.5 \mathrm{~m}, D$ is the equivalent distance from road segment to the reception point, $\mathrm{m}, a$ is the site parameter whose value depends on site conditions, $P$ is the percentage of soft ground cover within the segment, $\Delta \phi$ is the angle subtended by road segment relative to the receiver, degree, and $\Delta L_{\mathrm{s}}$ is the shielding adjustment for noise barrier, $\mathrm{dB}(\mathrm{A})$.

The model evaluation results indicated that noise prediction is more accurate at locations closer to the road carriageway where the environment of sound propagation is less complex. The model has an accuracy of $0.8 \mathrm{~dB}(\mathrm{~A})$ for traffic noise prediction at locations nearer to the road, and was accurate to $2.1 \mathrm{~dB}(\mathrm{~A})$ for locations within housing estate. Further, there were no errors in the prediction results observed in either set of conditions. The accuracy in predictive results of the adjusted model for China is comparable to those of the FHWA model whose predictive accuracy is $2.0 \mathrm{~dB}(\mathrm{~A})$.

\section{COMPARISON OF MODELS AND THE IDEAL MODEL}

In order to know the differences among the above models, comparison is made with respect to some salient attributes. Table 1 shows the comparison of models. There is a practical need for a model like the ideal one shown in the last column in meeting a range of needs. Capability is especially wanted for interrupted and complex flow, for predicting the effects of various traffic light cycles, traffic routings, pedestrian crossing locations, and other controls. The ideal model in comparison of models (Table 1) incorporates these attributes.

\section{RECENT DEVELOPMENTS}

The FHWA TNM model is an example of the trends toward more accurate physics in models and toward more realistic representations of the actual traffic flows. Another approach, by Cammarata et al. [23], 


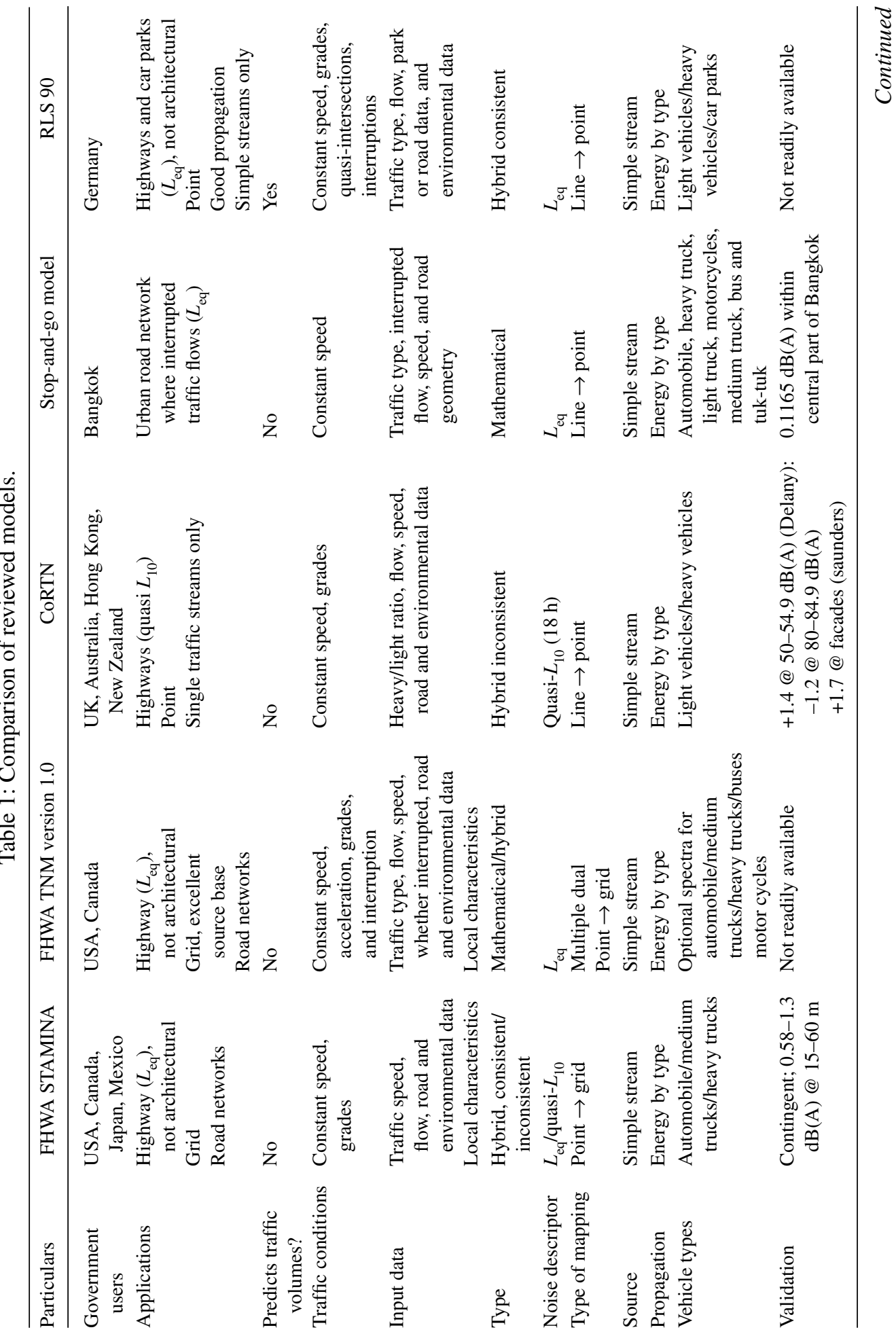




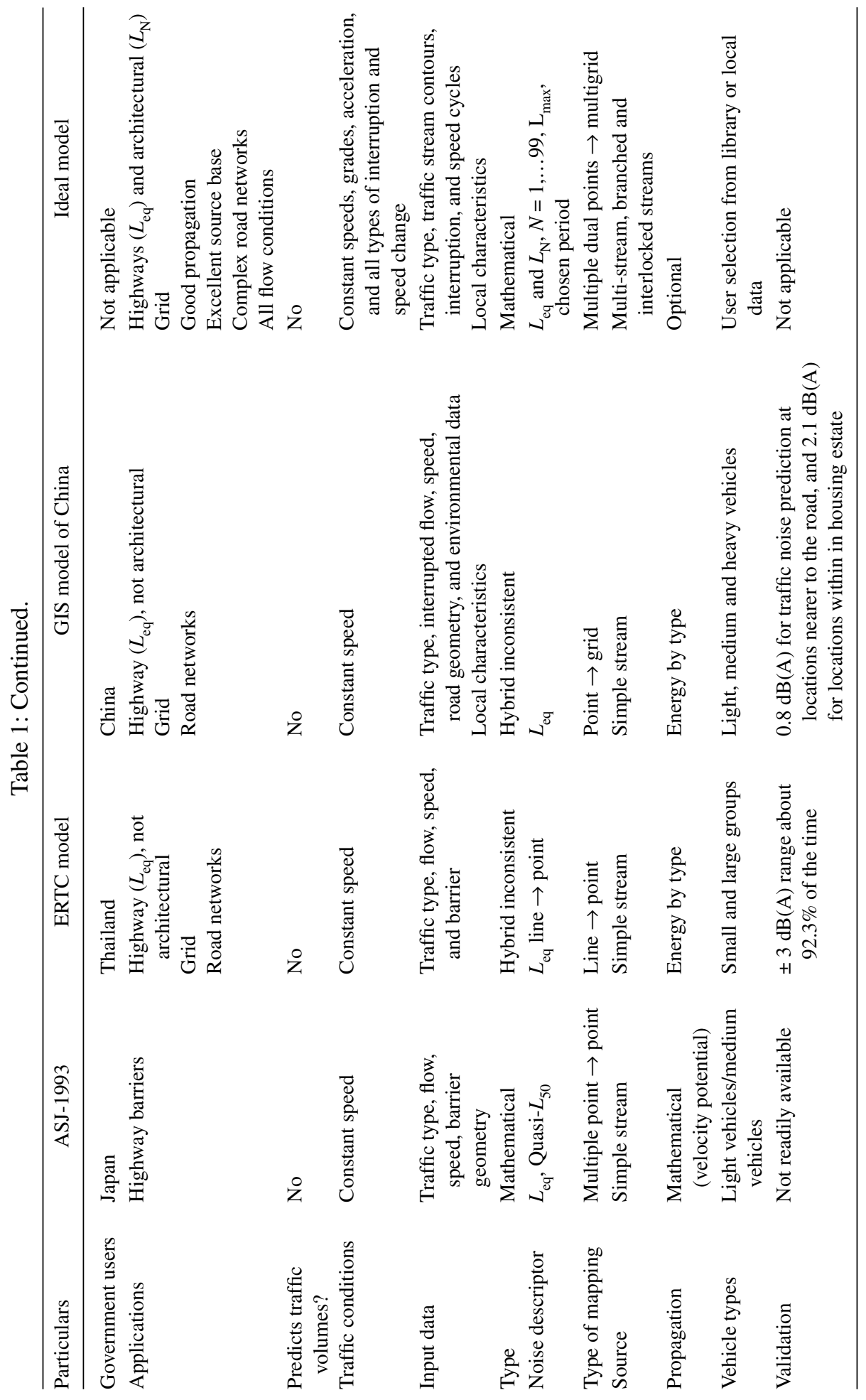


was to use a neural network scheme as a substitute for the linear regression in earlier models. He compared his results with Burgess [24], Josse [25], and Bertoni et al. [26], and found significant improvements for the neural network. In 1982, Samuels [27] developed an air pumping theory of tyre/road noise. His model comprised of four simple sources at both front and back of every tyre (32 sources) for an automobile.

\section{CONCLUSIONS}

Based on the critical review of the models, it can be concluded that within the range of validity, the models reviewed here meet the requirements of government regulations and many designers. Some models allow for other road vehicles as well as automobiles and trucks, and one includes car parks. All the models discussed here have acoustic energy descriptions usually explicit as $L_{\mathrm{eq}}$ or in two cases as a pseudo- $L_{10}$. The $L_{\text {eq }}$ models admit of easy corrections for interrupted flow, multiple streams, and multiple roads. The eight models reviewed here are designed to meet the requirements of roadway engineers. However, they do not meet the requirements of other users of traffic noise models. The ideal model is proposed to supply all the deficiency. Therefore, there is a need to develop an ideal model which satisfies all the constraints.

\section{ACKNOWLEDGMENTS}

The first author gratefully acknowledges the financial assistance provided by All India Council for Technical Education, New Delhi, under the scheme 'Career Award for Young Teachers' and the authorities of Adhiyamaan College of Engineering, Hosur, Tamil Nadu and P.E.S. College of Engineering, Mandya, Karnataka, for providing necessary facilities and support.

\section{REFERENCES}

[1] Nirjar, R.S., Jain, S.S., Parida, M. \& Katiyar, V.S., Study of transport related noise pollution in Delhi. J. Institution of Engineers (India), Environmental Engineering Division, 84, pp. 6-15, 2003.

[2] Bengang, Li., Shu-Tao \& Dawson, W., A GIS based road traffic noise prediction model. Applied Acoustics, 63(6), pp. 679-691, 2002.

[3] Campbell, S., A critical review of some traffic noise prediction models. Journal of Applied Acoustics, 62, pp. 271-287, 2001.

[4] Rathe, E.J., Note on two common problems of sound propagation. Journal of Sound and Vibration, 10, pp. 472-479, 1969.

[5] Steele, C.M., Report to the New South Wales Department of Public Works, CM. Steels \& Associates, 1985.

[6] Anon., Acoustics - Attenuation of Sound during Propagation Outdoors. Part 2: General Method of Calculation, ISO 9613-2, 1996.

[7] Maekawa, Z. Noise reduction by screens. Applied Acoustics, 1, pp. 57-73, 1968.

[8] Jain, S.S., Parida, M. \& Bhattacharya, C.C., Development of comprehensive highway noise model for Indian conditions. J. Indian Road Congress, 62(3), pp. 453-488, 2000.

[9] Barry, T.M. \& Reagan, J.A., FHWA Highway Traffic Noise Prediction Model, Report No. FHWA-RD-77-108, US DOT, FHWA, Office of Research, Office of Environmental Policy: Washington DC, USA.

[10] Anon., Highway Noise Measurements for Verification of Prediction Models (DOT-TSCOST-78-2/DOT-TSC-FHWA-78-1), Federal Highway Administration: Washington, 1978. 
[11] Junf, F.W., Blaney, C.T. \& Kazakov, A.L., Noise Emission Levels for Vehicles in Ontario, Transportation Research Record 105, 1996.

[12] Anderson, G.S., Menge, C.W., Rossano, C.F., Armstrong, R.E., Ronning, S.A., Flemming, G.G. \& Lee, C.S.Y., FHWA traffic noise model, version 1.0: introduction to its capacities and screen components. The Wall Journal, 22, pp. 14-17, 1996.

[13] Delany, M.E., Harland, D.G., Hood, R.A. \& Scholes, W.E., The prediction of noise levels $L_{10}$ due to road traffic. Journal of Sound and Vibration, 48(3), pp. 305-325, 1976.

[14] Anon., Calculation of Road Traffic Noise, United Kingdom Department of the Environment and Welsh Office Joint Publication/HMSO: London, 1975.

[15] Anon., Predicting Road Traffic Noise, United Kingdom Department of the Enivornment/HMSO: London.

[16] Samuels, S.E. \& Saunders, R.E., The Australian performance of the UK DoE traffic noise prediction method. Australian Road Research Board Conference, 1982.

[17] Weatherall, F., A Program for a programmable calculator for estimation of traffic noise by UK CoRTN procedure. Australian Acoustical Society 1985 Conference, 1985.

[18] Pamanikabud, P. \& Tharasawatpipat, C., Modeling of urban area stop-and-go model. Journal of Transportation Engineering, 125(2), 1999.

[19] Suksaard, T. \& Sukasem, P., Road traffic noise prediction model in Thailand. Applied Acoustics, 58(2), pp. 123-130, 1999.

[20] Koyasu, M., Method of Prediction and Control of Road Traffic Noise in Japan, Inter-noise 78: San Francisco, 1978

[21] Takagi, K. \& Yamamota, K. Calculation Methods for Road Traffic Noise Propagation Proposed by ASJ, Inter-noise: Yokohama, 1994.

[22] Rayleigh Baron (John William Strutt), The Theory of Sound, Dover Publications: New York, 1945 (work originally published in 1896).

[23] Cammarata, G., Cavalieri, S. \& Fichera, A., A neural network architecture for noise prediction. Neural Networks, 8(6), 1995.

[24] Burgess, M.A., Noise prediction for urban traffic conditions - related to measurements in the Sydney Metropolitan Area. Applied Acoustics, 10, pp. 1-7, 1977.

[25] Josse, R., Notions d'acoustique, ed. Eyrolles, Paris, France, 1972.

[26] Bertoni, D., Franchini, A. \& Magnoni, M., Centre Scientifique et Technique des Batiments, 1987.

[27] Samuels, S.E., The generation of tyre/road noise. Australian Road Research Board Research Report ARR 12, 1982. 